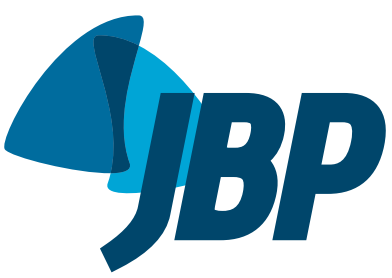

\section{COVID-19: o que aprendemos?}

\author{
Yeh-Li Ho1 (iD , Anna Miethke-Morais²
}

Em dezembro de 2019, foram relatados os primeiros casos de uma pneumonia de etiologia desconhecida na cidade de Wuhan, Hubei, China. No dia 7 de janeiro de 2020, confirmou-se de que se tratava de um novo coronavírus, que mais tarde recebeu o nome de SARSCoV-2, e a doença foi denominada COVID-19. (1) Passados quatro meses, a doença já foi detectada em mais de 185 países, acometendo quase três milhões de pessoas, com mais de 200 mil vidas perdidas. ${ }^{(2)}$

A escalada exponencial de casos levou governantes ao redor do mundo a adotar políticas restritivas de movimentação social com o objetivo de reduzir a taxa de transmissibilidade $\left(R_{0}\right)$ da doença, isto é, o número de contágios secundários a partir de um indivíduo infectado. $O$ valor $R_{0}$ de um agente infeccioso depende das características da doença - período de início de transmissão em relação ao início de sintomas, duração da transmissibilidade e formas de transmissão - e, em doenças de transmissão por via respiratória, da densidade populacional e do comportamento cultural. Usando modelos matemáticos, estudos observaram a redução da $R_{0}$ após o fechamento da cidade de Wuhan e a restrição da movimentação social. (3)

É importante ressaltar que o isolamento social visa a redução da transmissibilidade inter-humana com consequente achatamento da curva de casuística, porém, por si só, não impede a transmissão da doença em algum momento da vida enquanto houver a circulação do vírus na comunidade. Ainda assim, a medida de isolamento é importante para preparar os sistemas de saúde e permitir que esses prestem assistência suficiente e adequada aos enfermos. Modelos matemáticos desenhados para a Região Metropolitana de São Paulo usando um valor médio de $\mathrm{R}_{0}$ e a proporção de indivíduos hospitalizados em UTIs disponíveis na literatura até então estimaram que, nos primeiros de 30 dias após a confirmação do primeiro caso, haveria uma demanda de aproximadamente 5.300 leitos de UTI, $130 \%$ acima da capacidade real da região (estudo submetido à publicação). Dados similares foram observados em outros países, reforçando a importância do isolamento social para a redução da letalidade da doença. ${ }^{(4,5)}$

Visando ampliar a capacidade assistencial aos pacientes com COVID-19 no sistema público, diversas medidas foram tomadas pelos governantes de diferentes esferas, como aberturas de hospitais de campanha, ampliação da capacidade de leitos de UTI e parcerias com hospitais privados. Na cidade de São Paulo, por exemplo, o Hospital das Clínicas da Faculdade de Medicina da Universidade de São Paulo - um hospital de alta complexidade e o maior hospital público da América Latina - tornou-se um dos hospitais de referência para o atendimento de pacientes graves com COVID-19, destinando seu maior instituto, o Instituto Central, com capacidade de 900 leitos, ao atendimento exclusivo desses pacientes. A capacidade de leitos de UTI foi dobrada, passando a 200.

Essa alta demanda de leitos hospitalares decorre da diferença da COVID-19 de outras doenças respiratórias virais agudas. Enquanto a infecção por influenza A(H1N1) pdm09 possui curso evolutivo curto, com alguns pacientes necessitando de internação nos primeiros dias da doença e com tempo relativamente curto de hospitalização, ${ }^{(6,7)}$ pacientes infectados por SARS-CoV-2 apresentam deterioração clínica mais tardia e tempo de permanência hospitalar mais prolongado. ${ }^{(8,9)}$ Essas características provocam a necessidade de maior tempo de monitorização ambulatorial - especialmente entre os pacientes dos grupos de risco - e menor rotatividade de leitos hospitalares. Esses fatores podem contribuir para maior letalidade devido à falta de suporte hospitalar. Além disso, outros aspectos clínicos da COVID-19 também diferem dos de outras infecções respiratórias virais. Chamam a atenção a baixa frequência de sintomas respiratórios altos, o tempo prolongado de febre e mialgia, assim como a alta frequência de anosmia e/ou ageusia. ${ }^{(10,11)}$

Apesar dessas diferenças clínicas, a confirmação etiológica de SARS-CoV-2 depende de técnicas de biologia molecular, com sensibilidade variável de acordo com o momento da coleta e o material biológico analisado. ${ }^{(12)}$ Um estudo de Wang et al. observou uma sensibilidade de apenas $32 \%$ em swab de orofaringe e de $63 \%$ em swab de nasofaringe, amostras geralmente obtidas para confirmação diagnóstica. ${ }^{(13)}$ Além disso, o desempenho dos testes sorológicos também varia de acordo com a gravidade da doença e o momento da coleta, tornando seu uso limitado no momento de internação hospitalar. ${ }^{(14)}$ Diante desse cenário e somando-se o risco de exposição dos profissionais de saúde, diversas recomendações surgiram rapidamente na literatura médica em relação ao auxílio no diagnóstico, terapias de suporte (especialmente no contexto de terapia intensiva) e terapias antivirais específicas.

Entre as ferramentas de auxílio no diagnóstico, o padrão de alteração pulmonar na TC de tórax, com achados de infiltrado difuso de padrão em vidro fosco, foi a primeira estratégia adotada. Entretanto, é importante lembrarmos que diversas outras condições podem levar a esse achado radiológico, incluindo outras infecções pulmonares virais, como influenza. ${ }^{(15)}$ Outra recomendação de diversas sociedades médicas refere-se às estratégias de suporte 
na insuficiência respiratória aguda. A recomendação de intubação orotraqueal precoce visando a redução do risco de exposição dos profissionais da saúde foi amplamente divulgada. Entretanto, vale lembrar que essa medida pode levar à intubação desnecessária em pacientes com outros diagnósticos etiológicos; além disso, os pacientes são expostos aos riscos e complicações de sedação e ventilação mecânica prolongadas. Simultaneamente, estudos mais recentes mostraram que o uso de suportes ventilatórios não invasivos, como cateter nasal de alto fluxo e ventilação mecânica não invasiva, promove menores distâncias de propagação de partículas virais que aquelas com o uso de cateter nasal de oxigênio comum. ${ }^{(16,17)}$

Estratégias terapêuticas e antivirais também surgiram rapidamente na literatura, especialmente a transfusão de plasma de pacientes convalescidos, anticoagulação profilática e/ou plena e uso de medicamentos antivirais. Porém, nenhuma estratégia, até o momento da escrita deste manuscrito, tem evidência científica suficiente para ser recomendada na prática diária. É nesse aspecto que nós, médicos, precisamos ter cuidado com as nossas decisões, lembrando sempre dos riscos de complicações aos quais podemos submeter os nossos pacientes. Ressalta-se que o uso off-label de uma estratégia terapêutica é resguardado às situações de risco iminente de vida, e o paciente e/ou seus familiares precisam estar cientes da ausência de comprovação de eficácia. Ao mesmo tempo, cabe aos pesquisadores manter os critérios de rigor científico na elaboração e execução das pesquisas, assim como na divulgação dos resultados de estudos relacionados à COVID-19, evitando erros de interpretação da comunidade médica e da sociedade leiga. ${ }^{(18,19)}$

\section{REFERÊNCIAS}

1. Wang C, Horby PW, Hayden FG, Gao GF. A novel coronavirus outbreak of global health concern [published correction appears in Lancet. 2020 Jan 29]. Lancet. 2020;395(10223):470-473. https://doi. org/10.1016/S0140-6736(20)30185-9

2. Johns Hopkins University. Center for Systems Science and Engineering (CSSE) [homepage on the Internet]. Baltimore MD: CSSE; c2020 [cited 2020 Apr 26]. COVID-19 Dashboard. Available from: https://gisanddata.maps.arcgis.com/apps/opsdashboard/index. html/bda7594740fd40299423467b48e9ecf6

3. Zhu Y, Chen YQ. On a Statistical Transmission Model in Analysis of the Early Phase of COVID-19 Outbreak [published online ahead of print, 2020 Apr 2]. Stat Biosci. 2020;1-17. https://doi.org/10.1007/ s12561-020-09277-0

4. Demographic science aids in understanding the spread and fatality rates of COVID-19 [published online ahead of print, 2020 Apr 16. Proc Natl Acad Sci U S A. 2020;202004911. https://doi.org/10.1073/ pnas.2004911117

5. Tuite AR, Fisman DN, Greer AL. Mathematical modelling of COVID-19 transmission and mitigation strategies in the population of Ontario, Canada [published online ahead of print, 2020 Apr 8]. CMAJ 2020;cmaj.200476. https://doi.org/10.1503/cmaj.200476

6. Jain S, Kamimoto L, Bramley AM, Schmitz AM, Benoit SR, Louie J, et al. Hospitalized patients with $2009 \mathrm{H} 1 \mathrm{~N} 1$ influenza in the United States, April-June 2009. N Engl J Med. 2009;361(20):1935-1944 https://doi.org/10.1056/NEJMoa0906695

7. Bassetti M, Parisini A, Calzi A, Bobbio Pallavicini FM, Cassola G, Artioli $S$, et al. Risk factors for severe complications of the novel influenza A (H1N1): analysis of patients hospitalized in Italy. Clin Microbiol Infect. 2011;17(2):247-250. https://doi.org/10.1111/j.14690691.2010.03275.x

8. Guan WJ, Ni ZY, Hu Y, Liang WH, Ou CQ, He JX, et al. Clinical Characteristics of Coronavirus Disease 2019 in China. N Engl J Med. 2020;382(18):1708-1720. https://doi.org/10.1056/NEJMoa2002032

9. Grasselli G, Zangrillo A, Zanella A, Antonelli M, Cabrini L, Castelli A, et al. Baseline Characteristics and Outcomes of 1591 Patients Infected With SARS-CoV-2 Admitted to ICUs of the Lombardy Region, Italy [published online ahead of print, 2020 Apr 6]. JAMA 2020;323(16):1574-1581. https://doi.org/10.1001/jama.2020.5394

10. Fu L, Wang B, Yuan T, Chen $X$, Ao $Y$, Fitzpatrick T, et al. Clinical

characteristics of coronavirus disease 2019 (COVID-19) in China: A systematic review and meta-analysis [published online ahead of print, 2020 Apr 10]. J Infect. 2020;S0163-4453(20)30170-5. https:// doi.org/10.1016/j.jinf.2020.03.041

11. Yan CH, Faraji F, Prajapati DP, Boone CE, DeConde AS. Association of chemosensory dysfunction and Covid-19 in patients presenting with influenza-like symptoms [published online ahead of print, 2020 Apr 12]. Int Forum Allergy Rhinol. 2020;10.1002/alr.22579. https:// doi.org/10.1002/alr.22579

12. Tahamtan A, Ardebili A. Real-time RT-PCR in COVID-19 detection: issues affecting the results. Expert Rev Mol Diagn. 2020;20(5):453454. https://doi.org/10.1080/14737159.2020.1757437

13. Wang W, Xu Y, Gao R, Lu R, Han K, Wu G, et al. Detection of SARS CoV-2 in Different Types of Clinical Specimens [published online ahead of print, 2020 Mar 11]. JAMA. 2020;e203786. https://doi. org/10.1001/jama.2020.3786

14. Xiang $F$, Wang $X$, He X, Peng Z, Yang B, Zhang J, et al. Detection and Dynamic Characteristics in Patients with COVID-19 [published online ahead of print, 2020 Apr 19]. Clin Infect Dis. 2020;ciaa461.

15. Coppola M, Porto A, De Santo D, De Fronzo S, Grassi R, Rotondo A. Influenza A virus: radiological and clinical findings of patients hospitalised for pandemic H1N1 influenza. Radiol Med. 2011;116(5):706-719. https://doi.org/10.1007/s11547-011-0622-0

16. Li J, Fink JB, Ehrmann S. High-flow nasal cannula for COVID-19 patients: low risk of bio-aerosol dispersion [published online ahead of print, 2020 Apr 16]. Eur Respir J. 2020;2000892. https://doi. org/10.1183/13993003.00892-2020

17. Ferioli M, Cisternino $C$, Leo V, Pisani L, Palange $P$, Nava $S$ Protecting healthcare workers from SARS-CoV-2 infection: practical indications. Eur Respir Rev. 2020;29(155):200068. https://doi. org/10.1183/16000617.0068-2020

18. Kalil AC. Treating COVID-19-Off-Label Drug Use, Compassionate Use, and Randomized Clinical Trials During Pandemics [published online ahead of print, 2020 Mar 24]. JAMA. 2020;10.1001/ jama.2020.4742. https://doi.org/10.1001/jama.2020.4742

19. Habibi R, Burci GL, de Campos TC, Chirwa D, Cinà M, Dagron S et al. Do not violate the International Health Regulations during the COVID-19 outbreak. Lancet. 2020;395(10225):664-666. https://doi. org/10.1016/S0140-6736(20)30373-1 